\title{
An Infrared Image Target Segmentation Based on Improved Threshold Method
}

\author{
Manzeng Ma \\ College of Computer Science and Engineering, Cangzhou Normal University, Cangzhou 061001, Hebei, \\ China \\ Dan Liu* \\ College of Computer Science and Engineering, Cangzhou Normal University, Cangzhou 061001, Hebei, \\ China \\ *Corresponding author(E-mail: 9527a@163.com) \\ Ruirui Zhang \\ College of Computer Science and Engineering, Cangzhou Normal University, Cangzhou 061001, Hebei, \\ China
}

Received: February 1, 2021. Revised: July 9, 2021. Accepted: July 28, 2021. Published: July 30, 2021.

\begin{abstract}
In recent years, infrared images have been applied in more and more extensive fields and the current research of infrared image segmentation and recognition can't satisfy the needs of practical engineering applications. The interference of various factors on infrared detectors result in the targets detected presenting the targets of low contrast, low signal-to-noise ratio (SNR) and fuzzy edges on the infrared image, thus increasing the difficulty of target detection and recognition; therefore, it is the key point to segment the target in an accurate and complete manner when it comes to infrared target detection and recognition and it has great importance and practical value to make in-depth research in this respect. Intelligent algorithms have paved a new way for infrared image segmentation. To achieve target detection, segmentation, recognition and tracking with infrared imaging infrared thermography technology mainly analyzes such features as the grayscale, location and contour information of both background and target of infrared image, segments the target from the background with the help of various tools, extracts the corresponding target features and then proceeds recognition and tracking. To seek the optimal threshold of an image can be seen as to find the optimum value of a confinement problem. As to seek the threshold requires much computation, to seek the threshold through intelligent algorithms is more accurate. This paper proposes an automatic segmentation method for infrared target image based on differential evolution (DE) algorithm and OTSU. This proposed method not only takes into consideration the grayscale information of the image, but also pays attention to the relevant information of neighborhood space to facilitate more accurate image segmentation. After determining the scope of the optimal threshold, it integrates DE's ability of globally searching the optimal solution. This method can lower the operation time and improve the segmentation efficiency. The simulation experiment proves that this method is very effective.
\end{abstract}

Keywords - Infrared Image; Image Target Segmentation; Differential Evolution Algorithm; OTSU Threshold Segmentation.

\section{INTRODUCTION}

$\mathrm{A}$ ny object in the normal state, its surface will emit infrared, infrared energy increases with the temperature. The infrared thermal imager can obtain the thermal infrared image of the object by receiving the thermal radiation emitted by the detected object without touching the object. At present, infrared thermal imaging technology is widely used in many scientific research fields: missile, rocket, composite material detection, heat conduction research, mechanical fault diagnosis and so on [1]. Infrared is an electromagnetic wave with the same essence as radio wave and visible light. The discovery of infrared ray is a leap in human understanding of nature. The technology of using a special electronic device to convert the temperature distribution on the object surface into an image visible to human eyes and display the temperature distribution on the object surface in different colors is called infrared thermal imaging technology; In essence, it is the thermal image distribution of the infrared radiation of each part of the measured target object. Image segmentation is the premise and foundation to solve all image problems, and the result of image segmentation is directly related to the effect of later image processing. Therefore, the segmentation of infrared image has always been a hot issue for people to study, and it has a certain practical significance to conduct in-depth research on this topic. Compared with visible images, infrared images generally have some disadvantages, such as poor contrast between target and background, blurred edge, high noise and so on. Firstly, the infrared image has the disadvantages of low signal-to-noise 
ratio and contrast, and the edge and texture features are not obvious; secondly, because the infrared imaging system is vulnerable to atmospheric radiation, and the infrared detector also has the noise that can't be eliminated, which makes the target signal in the infrared image is very weak when the target is far away, and the background of the target is complex Clutter, the target is likely to be submerged in the complex background and noise, these factors lead to the target detection and tracking problems become more difficult [2]. Image segmentation is to segment an image into several segments or regions, and extract meaningful and interesting parts, which is a key step in image processing and image analysis [3]. Image segmentation is a basic field in image analysis. Firstly, segmentation is the key step of image preprocessing, which is the basis of image processing techniques such as detection and feature extraction. On the other hand, image segmentation can also be applied to image compression and video processing, which need to save memory space [4]. The inherent particularity of infrared target image makes it more difficult to segment, which is mainly reflected in that the infrared imaging is heat source imaging, the target and boundary in the image are blurred; the target itself has no obvious shape, size, texture and other information to use; the imaging area of the target is small, often accompanied by weak signal intensity, so the target segmentation should be carried out under the condition of low signal-to-noise ratio Because of the complexity, the contrast of the infrared vehicle target image is low and the segmentation is difficult [5].

Since the infrared imaging guidance technology has been studied in the United States in the 1970s, infrared target segmentation algorithm has started developing rapidly. Reed et al has further simplified the matched filter so as to detect the moving target at the same speed of the filter at the circumstance of low signal-to-noise ratio (SNR) [6]. Blostein et al has applied the hypothesis test method of multi-segmentation and achieved the tracking of moving targets [7]. Yu Jiaxue et al has proposed to combine CA with OTSU and applied it to the study of the target recognition and diagnosis of electrical equipment [8]. After over 40-year in-depth research, scholars have come up with dozens of infrared target segmentation methods, mainly including threshold segmentation, edge segmentation and region segmentation. Among conventional image segmentation methods, threshold segmentation has drawn extensive attention due to its simple operations, small storage space, fast processing speed and easy operation [9]. Threshold-based image segmentation methods include OTSU, the minimum error method (MEM), KSW, the maximum crossover entropy (MCE) and so on. The shortcoming of threshold segmentation technology lies in its sensitivity to noises; so it results in bad segmentation to noisy image. OTSU makes uses of the variance of pixel region and calculate the optimal threshold by seeking the maximum between-class variance. When the target variance and background variance reach the maximum, it indicates the maximum difference between the regions in the segmentation result and the best segmentation effect. Lee has discussed the defects of OTSU and its application scenarios [10]. Based on the analysis of OTSU's flaws, Hou has brought forward a threshold method based on the minimum between-class variance, which has introduced the probabilities two classes [11]. Ng has proposed to revise the histogram by the use of edge information against the unobvious bimodal distribution of OTSU in the image histogram so as to highlight and valley bottom features [12]. Xiong has raised a threshold algorithm which replaced the minimum between-class variance with the minimum between-class absolute difference as the segmentation criterion, and calculate the areas of both the background region and the target region and make full use of the image information [13]. However, according to the current research, there is no universal method which can adapt to the study of different images and nor is there universal evaluation standard formulated around the world to handle such kind of problems. Meanwhile, as images are greatly uncertain, it is very hard to identify the segmentation method suitable for all methods; so people are still exploring. Differential evolution algorithm (DE) was presented by R. Stom and K.Price in 1995 and it was proven the fastest evolution algorithm in the first IEEE in 1996 [14]. Moreover, DE has exceeded several other famous random algorithms in terms of convergence speed and stability. In addition, as DE is easy to comprehend and simple to implement, it has attracted much attention and been widely applied since its emergence [15]. This paper has combined DE with OTSU and got the auto infrared target image segmentation method with extraordinary performance.

This paper first introduces the background and significance of infrared image research and the research status of image segmentation at home and abroad. Then the image segmentation, threshold segmentation and differential evolution algorithm are analyzed. On the basis of the above, this paper studies the relevant mathematical formula of threshold problem, how to improve DE algorithm, proposes the improvement strategy of crossover operator and mutation operator, and the evaluation of image segmentation effect, and puts forward the steps and process of optimizing infrared image threshold segmentation based on de and Otsu. Finally, the test experiment and result analysis verify the effectiveness of the proposed algorithm.

\section{OTSU IMAge SEgmentATION}

OTSU, also known as the maximum between-class variance threshold selection method, is an adaptive threshold determination method [16]. It divides the image into two parts: the background and the target according to the grayscale characteristic of the image. The bigger the between-class variance between background and target is, the greater the difference of the two parts which constitute the image is. The difference between the two parts will be reduced when part of the target is wrongly separated as the background or vice versa. Therefore, the segmentation that maximizes the between-class variance means the minimum probability of wrong separation [17] [18].

Assume that $X$ is an image with $L$ levels of grayscale and the $i$ th level has $N_{i}$ pixels with the value of $i$ within the scope of $0 \sim L-1$. Then the total number of pixels of the image is 


$$
N=\sum_{i=0}^{L-1} N i
$$

The probability for the $i$ th level to appear is

$$
P_{i}=\frac{N_{i}}{N}
$$

In OTSU, the threshold $k$ divides all pixels into two classes: the target $C_{0}$ and the background $C_{1}$. Among them, the level of pixel grayscale of Class $C_{0}$ is $0 \sim k-1$ and that of Class $C_{1}$ is $k \sim L-1$.

The total average grayscale of the image is

$$
\mu=\sum_{i=0}^{L-1} i P_{i}
$$

The percentage of Class $C_{0}$ pixels occupying the total area is

$$
\omega_{0}=\sum_{i=0}^{k-1} P_{i}
$$

The percentage of Class $C_{1}$ pixels taking up the total area is

$$
\omega_{1}=1-\omega_{0}
$$

The average grayscale of Class $C_{0}$ is

$$
\mu_{0}=\mu_{0}(k) / \omega_{0}
$$

The average grayscale of Class $C_{1}$ is

$$
\mu_{1}=\mu_{1}(k) / \omega_{1}
$$

Where,

$$
\begin{gathered}
\mu_{0}(k)=\sum_{i=0}^{k-1} i P_{i} \\
\mu_{1}(k)=\sum_{i=k}^{L-1} i P_{i}=1-\mu_{0}(k)
\end{gathered}
$$

The formula for the maximum between-class variance is

$$
\delta^{2}(k)=\omega_{0}\left(\mu-\mu_{0}\right)^{2}+\omega_{1}\left(\mu-\mu_{1}\right)^{2}
$$

Make $k$ change among $0 \sim L-1$ and calculate the between-class variance $\delta^{2}(k)$ under different $k$. The $k$ which maximizes $\delta^{2}(k)$ is the optimal threshold to be found [19].

\section{IMPROVEMENT STRATEGY OF DIFFERENTIAL EVOLUTION ALGORITHM}

In the random vector difference method, the variation of each individual depends on the vector difference of two random individuals. The optimal solution plus random vector difference method is adopted. Each individual is determined by the current optimal solution and distributed in the neighborhood of the current optimal solution [20]. The information of the optimal individual of the current optimal population is used to speed up the search speed, but at the same time, if the population distribution density is high, it may lead to computational complexity, compared with the method of optimal solution plus random vector, the difference method of optimal solution and random vector can reduce the risk of falling into local optimal solution. When the vector deviation is large, the individual variation intensity is high; on the contrary, the individual variation intensity is low. Therefore, if the population distribution density is high, the individual variation intensity is low [21] [22].

\section{A. Basic Differential Evolution Algorithm}

(1) Population initialization

Randomly generate the initial population $\mathrm{X}(0)=\left\{x_{1}^{0}, x_{2}^{0}, \ldots, x_{\mathrm{NP}}^{0}\right\}$ in the solution space of the problem. $x_{i}^{0}=\left[x_{i, 1}^{0}, x_{i, 2}^{0}, \ldots, x_{i, D}^{0}\right]$ is used to represent the $i$ th individual solution. Each component of an individual can be generated according to the following formula:

$$
x_{i, j}^{0}=x_{j, \min }+\operatorname{rand}\left(x_{j, \max }-x_{j, \min }\right)
$$

Where $x_{j, \max }$ and $x_{j, \min }$ are the upper and lower bounds of the $j$ th dimension of the solution space [23].

(2) Mutation operation

For any one target vector $x_{i}$ in the parent population, generate the mutation vector $v_{i}$ according to the following formula:

$$
v_{i}=x_{r 1}+F \times\left(x_{r 2}-x_{r 2}\right), i=1,2, \ldots, N P
$$

Where $\left\{x_{r 1}, x_{r 2}, x_{r 3}\right\}$ are three different individuals randomly selected from the parent population and $r 1 \neq r_{2} \neq r_{3} \neq i$.It is clear that the population size shall satisfy $N P \geq 4$. The scaling factor $F$ is a real constant factor between $[0$, 
2] and it is used to control the influence of the differential vector $\left(x_{r 2}-x_{r 3}\right)$.

\section{(3) Crossover operation}

In $\mathrm{DE}$ calculation, the subjects to have crossover operation are the individuals in the parent generation and the new individuals obtained after differential mutation. Although this method seems to have no information exchange among individuals, the new individuals come after different mutation and they have preserve the information of other individuals in the population; so the crossover operators in DE also has the mechanism of information exchange among individuals [24].

The purpose of crossover operation is to improve the diversity of individuals in the population through random recombination of every dimension of components of mutation vector $v_{i}$ and target vector $x_{i}$. The algorithm generates the new crossover vector $u_{i}=\left[u_{i, 1}, u_{i, 2}, \cdots, u_{i}, D\right]$ through the formula below:

$$
\begin{aligned}
& u_{i, j}=\left\{\begin{array}{l}
v_{i, j}, r \text { and } b \leq C R \text { and } j=r \text { and } j \\
x_{i, j}, r \text { and } b>C R \text { and } j \neq r \text { and } j
\end{array}\right. \\
& i=1, \ldots, N P, j=1, \ldots, N P
\end{aligned}
$$

$r$ and $b$ is a random number in [0,1]; $C R$ is a constant in $[0,1]$ and the bigger the value of $C R$, the more probable to have crossover; and $r$ and $j$ is an integer randomly selected in [1, $D$ ], which ensures that $u_{i}$ can obtain at least 1 element from $v_{i}$ in order to make sure the generation of new individuals and avoid stagnation of population evolution.

(4) Selection operation

When and only when the fitness value of the new vector individual $u_{i}$ is better than that of the target vector individual $x_{i}, u_{i}$ will be accepted by the population; otherwise, $x_{i}$ will still be preserved in the population of the next generation and implement mutation and crossover operations as the target vector in the next iteration. Assume that the optimization problem is $\min f(x)$, then the selection operation can be described with the following formula:

$$
x_{i}^{t+1}=\left\{\begin{array}{l}
u_{i}, f\left(u_{i}\right)<f\left(x_{i}^{t}\right) \\
x_{i}^{t}, \text { else }
\end{array}\right.
$$

The selection operation in DE means that the individuals in the parent generation compete with the newly-generated candidate individuals one by one and only the fittest survive so that the individuals in the offspring generation are always better than or equal to those in the parent generation so as to make the population always evolve toward the optimal solution [25]. Fig.1 is the diagram of differential evolution algorithm.

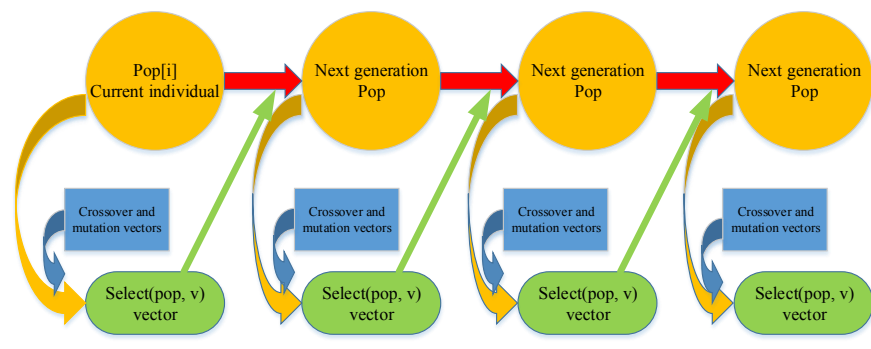

Fig 1. Diagram of differential evolution algorithm

\section{B. Improvement Strategy of Crossover Operator}

In order to improve the optimization performance of $\mathrm{DE}$, the progressively increasing crossover probability strategies of crossover probability factor is are adopted and their forms are clarified as follows:

The $1^{\text {st }}$ is exponential increase strategy, i.e.

$$
C R=C R_{\min }+\left(C R_{\max }-C R_{\min }\right) \exp \left(-a\left(1-t / T_{\max }\right)^{b}\right)
$$

The $2^{\text {nd }}$ is the parabola going downward, i.e.

$$
C R=\left(C R_{\min }-C R_{\max }\right)\left[\left(\frac{t}{T_{\max }}\right)^{2}-2 \frac{t}{T_{\max }}+1\right]+C R_{\max }
$$

The $3^{\text {rd }}$ is the parabola going upward, i.e.

$$
C R=\left(C R_{\max }-C R_{\min }\right)\left(\frac{t}{T_{\max }}\right)^{2}+C R_{\min }
$$

The $4^{\text {th }}$ is the invariant crossover probability factor $C R$, i.e.

$$
C R=C R_{\min }+\frac{t\left(C R_{\max }-C R_{\min }\right)}{T_{\max }}
$$

Among them, $t$ is the number of the current iterations, $T_{\max }$ is the maximum number of iteration, and parameters $C R_{\min }=0.1, C R_{\max }=0.9$. The above crossover probability can better balance the global search ability and local search ability to make the algorithm fast converged to the optimal solution [26]. DE uses the swarm intelligence model generated by the cooperation and competition among individuals in the group to guide the optimization search. Different from other evolutionary computation, differential evolutionary computation retains the global search strategy based on population, and adopts real number coding, differential mutation operation and one-to-one competitive survival strategy to reduce the complexity of evolutionary operation. Differential evolutionary computation has strong global convergence ability and robustness due to its unique evolutionary operation, which is very suitable for solving some optimization problems in complex environments. 


\section{Improvement Strategy of Mutation Operator}

In the initial phase, it has strong global search ability and can find as many global optimal points as possible while in the later phase, it shall have strong local search ability to improve the accuracy and convergence speed of the algorithm. For this purpose, it has introduced the simulated annealing strategy and set $\lambda$ as the annealing factor, as shown in Formula (19).

The mutation operation formula is

$$
v_{i}^{t}=\lambda x_{r 1}^{t}+(1-\lambda) x_{b e s t}^{t}+F\left(x_{r 2}^{t}-x_{r 3}^{t}+x_{r 4}^{t}-x_{r 5}^{t}\right)
$$

Where $\lambda \in[0,1]$. In the search process, $\lambda$ gradually changes from 1 to 0 , making the weight of $x_{r 1}$ gradually decrease and that of $x_{\text {best }}$ gradually increase so as to ensure that the algorithm not only has strong global search, but also has fast convergence speed and search accuracy [27] [28].

\section{Implementation Steps of Improved DE Algorithm}

Step 1: Set the basic parameters, including the population size $N$, the maximum number of iterations $T_{\max }$, the upper and lower bounds of scaling factor $F_{\max }, F_{\min }$, and the upper and lower bounds of the crossover probability $C R_{\min }, C R_{\max }$.

Step 2: Set the iteration counter $t=0$.

Step 3: According to Formulas (15),(16),(17) and (18), carry out mutation, crossover and selection on all individuals in DE population.

Step 4: Judge whether individuals have the stagnation phenomenon; if yes, implement mutation operation according to Formula (19).

Step 5: Update the iteration counter $t=t+1$ and record the optimal individual in the entire population. If the accuracy requirement is met or the entire evolution has reached the maximum number of iterations, stop the algorithm; otherwise, turn to Step 3.

\section{Determination of the Optimal Segmentation THRESHOLD COMBINING DE ALGORITHM AND OTSU}

\section{A. Selection of Region of Interest}

The so-called Region of Interest (ROI) means the region which contains the target; without any doubt, the smaller the region is, the less calculation there will be in the follow-up steps. The distance between the target and the observer can be measured by installing laser rangefinder in the vehicles and according to this distance, the minimum and maximum heights and widths can be estimated. Laser ranging is an instrument that uses laser to accurately measure the target distance. During operation, a very fine laser beam is emitted to the target, and the photoelectric element receives the laser beam reflected by the target. The timer measures the time from transmitting to receiving the laser beam and calculates the distance from the observer to the target. Besides, for infrared images, it is usually hypothesized that the target has higher brightness than the background; so make $H i s(g)$ represent the histogram of the infrared image, where g means the grayscale value of the pixels in the image and the global threshold can be determined according to P-Tile thresholding method:

$$
T_{\text {global }}=\arg \max _{t} \sum_{g=0}^{t} H i s(g) \leq\left(H W-H_{\min } W_{\min }\right)
$$

In Formula (20), $H_{\min }$ and $W_{\min }$ represent the minimum height and width of the target determined by the priori knowledge respectively while $H$ and $W$ are the height and width respectively of the original image. After the determined global threshold $T_{\text {global }}$ binarize the original infrared image, it makes the binary image after being thresholded through " 8 neighborhood" method and select the biggest region as the region of reference $R_{r}$. In ROI, the rest other than $R_{r}$ are all background region $R_{b}$. Then according to $R_{r}$ as well as the maximum height $H_{\text {max }}$ and width $W_{\max }$ of the given target, it determines the ROI.

The selection of region, the subsequent image processing can only be conducted on the ROI so as to greatly enhance the operation speed.

\section{B. Adaptive Fuzzy Enhancement of Infrared Target Image}

Image enhancement is to process the original image through certain processing methods to get the image of better quality. It makes the unclear image clear or highlights certain features we are interested in to improve the visual effect of the image or to facilitate other image processing.

The image can be represented by the two-tuples $(i, j)$ formed by $f(x, y)$ and $g(x, y)$. If $(i, j)$ appears $f(i, j)$ times, then for an image with a size of $M \times N$, the corresponding joint probability density $p(i, j)$ is

$$
p(i, j)=f(i, j) /(M \times N)
$$

Where $O<i, j<(L-1)$. Then

$$
\sum_{i=0}^{L-1} \sum_{j=0}^{L-1} p_{i j}=1
$$

Make the 2D vector $(S, T)$ as the threshold and the $2 \mathrm{D}$ histogram of the image can be divided into 4 regions. According to homomorphism, the grayscale value of pixel is close to the average grayscale value of neighborhood at the target and background while in the boundary field of the target and background, the difference between the above two values is quite big. Therefore, the pixels in the target and background will appear in the surrounding regions of the diagonal line. Region $A$ represents the background and Region $B$ presents the target and $C$ and $D$ far from the diagonal line represent the possible edges and noises. 
Make two classes $C_{0}$ and $C l$ represent the background and the target and the probability distributions for them to appear are respectively:

$$
\begin{aligned}
& W_{0}=P_{r}\left(C_{0}\right)=\sum_{i=0}^{S} \sum_{j=0}^{T} P_{i j}=W_{0}(S, T) \\
& W_{1}=P_{r}\left(C_{1}\right)=\sum_{i=S+1}^{L-1} \sum_{j=S+1}^{L-1} P_{i j}=W_{1}(S, T)
\end{aligned}
$$

Where $W_{0}$ means the probability for the background to occur and $W_{1}$ is the probability for the target to occur. The corresponding mean vectors to the background and target are

$$
\begin{aligned}
& u_{0}=\left(u_{0 i} u_{0 j}\right)^{T}=\left[\sum_{i=0}^{S} \sum_{j=0}^{T} i p_{i j} / W_{0}(S, T) \sum_{i=0}^{S} \sum_{j=0}^{T} j p_{i j} / W_{0}(S, T)\right]^{T} \\
& u_{1}=\left(u_{1 i} u_{1 j}\right)^{T}=\left[\sum_{i=S+1}^{L-1} \sum_{j=T+1}^{L-1} i p_{i j} / W_{1}(S, T) \sum_{i=S+1}^{L-1} \sum_{j=T+1}^{L-1} j p_{i j} / W_{1}(S, T)\right]^{T}
\end{aligned}
$$

As the probability of the diagonal line far away from the histogram can be ignored, then $W_{0}+W_{1} \approx 1$ and the total mean $u_{z}$ can be represented as

$$
u_{z}=\left(u_{z i} u_{z j}\right)^{T}=\left[\sum_{i=0}^{L-1} \sum_{j=0}^{L-1} i p_{i j} \sum_{i=0}^{L-1} \sum_{j=0}^{L-1} j p_{i j}\right]^{T}=W_{0} u_{0}+W_{1} u_{1}
$$

Define a discrete measurement matrix between the target and the background as follows:

$$
\begin{aligned}
\sigma B & =\sum_{k=0}^{L} P_{r}\left(C_{k}\right)\left[\left(u_{k}-u_{z}\right)\left(u_{k}-u_{z}\right)^{T}\right] \\
& =W_{0}\left[\left(u_{0}-u_{z}\right)\left(u_{0}-u_{z}\right)^{T}\right]+W_{1}\left[\left(u_{1}-u_{z}\right)\left(u_{1}-u_{z}\right)^{T}\right]
\end{aligned}
$$

Use the trace $\operatorname{tr} \sigma B$ of matrix $\sigma B$ as the distance measurement function between the target and the background:

$$
\begin{aligned}
\operatorname{tr} \sigma B & =W_{0}\left[\left(u_{0 i}-u_{z i}\right)^{2}+\left(u_{0 j}-u_{z j}\right)^{2}\right]+W_{1}\left[\left(u_{1 i}-u_{z i}\right)^{2}+\left(u_{1 j}-u_{z j}\right)^{2}\right] \\
& =\left[W_{0}(S, T) u_{z i}-u_{z i}(S, T)\right)^{2}+\left(W_{0}(S, T) u_{z j}\right. \\
& \left.\left.-u_{j}(S, T)\right)^{2}\right] /\left[W_{0}(S, T)\left(1-W_{0}(S, T)\right)\right]
\end{aligned}
$$
$W_{0}(S, T), u_{i}(S, T), u_{1}(S, T)$ and among them,

$$
W_{0}(S, T)=\sum_{i=0}^{S} \sum_{j=0}^{T} p_{i j} ; u_{i}(S, T)=\sum_{i=0}^{S} \sum_{j=0}^{T} i p_{i j} ; u_{j}(S, T)=\sum_{i=0}^{S} \sum_{j=0}^{T} j p_{i j}
$$

The threshold $\left(S_{0}, T_{0}\right)$ of 2D OTSU image segmentation is taken when $\operatorname{tr} \sigma B(S, T)$, namely

$$
\operatorname{tr} \sigma\left(S_{0}, T_{0}\right)=\operatorname{Max}\{\operatorname{tr} \sigma B(S, T)\} \quad 0 \leq S, T \leq 1
$$

The downside of using OTSU in image segmentation is the large amount of computation. For any $(S, T)$, the three variables $W_{0}(S, T), u_{i}(S, T), u_{j}(S, T)$ in the measurement function need to seek their cumulative sum and traverse all $S$ and $T$. The complexity of the calculation is approximately $O\left(L^{4}\right)$. Therefore, this paper searches the scope of the optimal threshold for ROI and makes use of DE's (Differential Evolution) to seek global optimal solution to reduce the calculation time and improve the segmentation efficiency.

C. Determination of the Range of the Optimal Threshold of ROI Segmentation

If the scope of the optimal threshold of ROI can be determined, to search the optimal segmentation threshold $\left(S_{0}, T_{0}\right)$ with DE will greatly accelerate the speed of DE.

Assume that the size of an image is $M \times N$ and the grayscale values include $0,1, \cdots, L-1$. Then the initial threshold is

$$
T_{0}=\frac{\sum_{x=0}^{M-1} \sum_{y=0}^{N-1} f(x, y)}{K}
$$

Where $f(x, y)$ is the grayscale value of point $(x, y)$ and $K=M \times N$ is the total number of pixels.

The initial threshold $T_{0}$ segments the image into two parts. According to characteristics of infrared image, the target usually has higher brightness than the background. Assume that the part bigger than $T_{0}$ is the target region $B$ and calculate $B$ 's mean grayscale, i.e.

$$
T_{B_{1}}=\frac{\sum \sum f_{B_{1}}(x, y)}{N_{B_{1}}}
$$

Where $f_{B_{1}}(x, y)$ is the grayscale value of all points in Region $B$ after being segmented by the initial threshold $T_{0}$ and $N_{B_{1}}$ is the total number of pixels in Region $B$. Given the actual complex scenes of infrared image, the grayscale of the background may be higher than that of certain target. In this way, to segment the image with the initial threshold may misjudge part of bright background as the target; in other words, the grayscale value of the target must be higher than the initial threshold $T_{0}$. So $T_{0}$ can be set as the lower bound of the threshold, which can ensure the completeness of the target segmentation. As for the determination of the upper bound of the threshold, it is hoped to use the image after being segmented by the initial threshold $T_{0}$ to increase the percentage of the target and the mean grayscale $T_{B}$ of the segmented region. 
Through the practical experiment, it can be seen that the points with grayscale higher than $T_{B}$ must be the target points. Therefore, the upper bound of the threshold can be determined as $T_{B}$. Till now, the scope of the optimal threshold is $\left[\begin{array}{ll}T_{0} & T_{B}\end{array}\right]$.

\section{EXPERIMENT TEST AND ANALYSIS}

This paper uses two objective evaluation criteria: F-Measure (FM) [29] and Localization error (LE) [30] to compare the segmentation effects by 3 algorithms. Among them, the higher the FM, the better the segmentation effect while the smaller the LE, the fewer positioning error and the better the segmentation effect.

Assume that an image only contains 1 target region and the rest are background. Let set $S$ represents the set of the pixels in the target region obtained by the segmentation methods, set $\mathrm{T}$ represents the set of pixels in the target region of the reference image, and I means the set of all pixels of the entire image. Then the following 4 measures are defined:

True positive: $T P=S \bigcap T$; False positive: $F P=S-T$

False negative: $F N=S-T$; True negative: $T N=I-T-S$

FM is the harmonic mean of segmentation accuracy and recall rate. The higher its value, the higher the segmentation accuracy. LE calculates the distance between the pixels wrongly classified and the nearest pixel in the reference image. The smaller the value, the lower the segmentation error. The specific calculation formulas are as follows:

$$
\begin{aligned}
& F M=2 T P /(2 T P+F P+F N) \\
& L E=(F N+F P) /(T P+T N+F P+T N)
\end{aligned}
$$

In this experiment, 4 images are selected and Contour extraction algorithm, Otsu algorithm and the proposed algorithm are adopted. After the experiment, the segmentation effects by these algorithms are compared with the data results seen in Table 1 and Table 2.

Table 1 Comparison of FM of Segmentation Results of Algorithms

\begin{tabular}{|c|c|c|c|}
\hline Infrared image & $\begin{array}{c}\text { Contour } \\
\text { extraction } \\
\text { method }\end{array}$ & Otsu & $\begin{array}{c}\text { Our } \\
\text { proposed } \\
\text { algorithm }\end{array}$ \\
\hline Aircraft & 0.7859 & 0.7783 & 0.8924 \\
\hline Black bear & 0.7448 & 0.6611 & 0.8356 \\
\hline Panda & 0.8272 & 0.8025 & 0.8451 \\
\hline Horses & 0.8066 & 0.8332 & 0.8587 \\
\hline
\end{tabular}

Table 2 Comparison of LE of Segmentation Results of Algorithms

\begin{tabular}{|c|c|c|c|}
\hline Infrared image & $\begin{array}{c}\text { Contour } \\
\text { extraction } \\
\text { method }\end{array}$ & Otsu & $\begin{array}{c}\text { Our } \\
\text { proposed } \\
\text { algorithm }\end{array}$ \\
\hline Aircraft & 0.0426 & 0.0517 & 0.0332 \\
\hline Black bear & 0.0573 & 0.1013 & 0.0506 \\
\hline Panda & 0.0275 & 0.0345 & 0.0211 \\
\hline Horses & 0.0429 & 0.0442 & 0.0368 \\
\hline
\end{tabular}

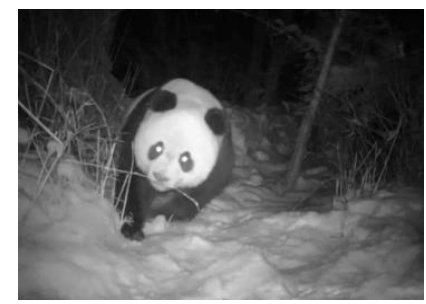

(a) Original image

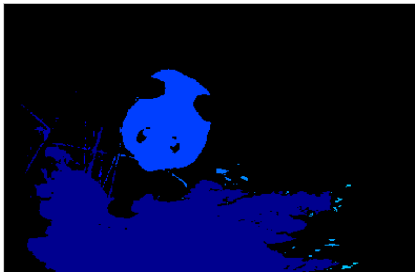

(c) Label image
As shown from the comparison of FM and LE by 3 algorithms in Table 1 and Table 2, the proposed algorithm in this paper has the best segmentation effect. The segmentation results on 4 test images by the proposed image are demonstrated in Fig.2-Fig.4 below.

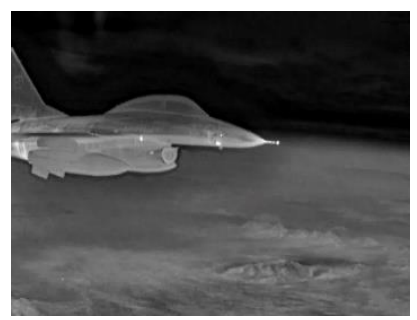

(a) Original image

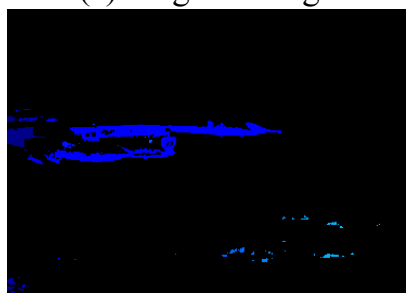

(c) Label image

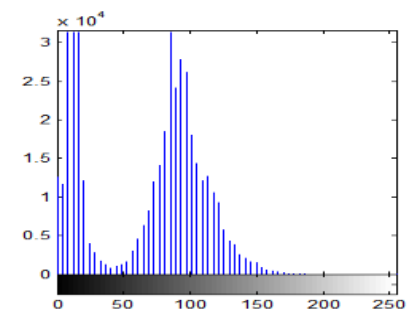

(b) Histogram

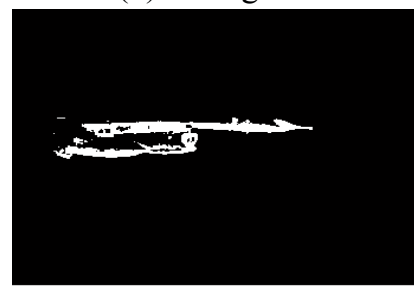

(d) Image segmentation

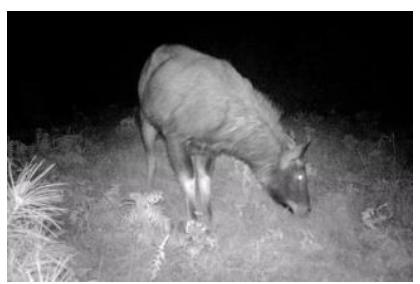

(a) Original image

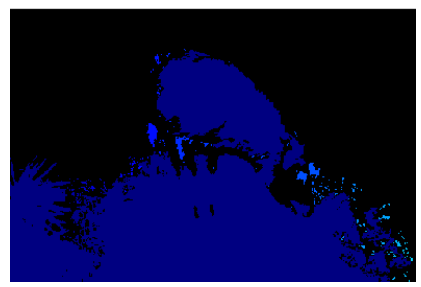

(c) Label image

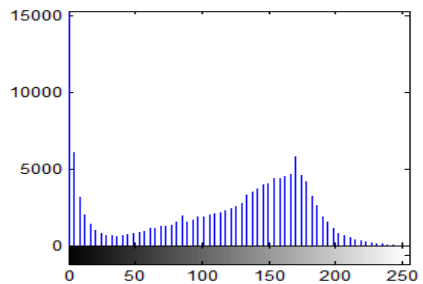

(b) Histogram

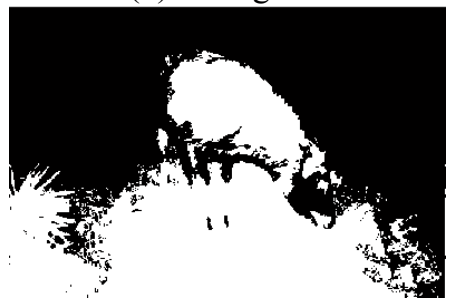

(d) Image segmentation
Fig 3. Segmentation effect of Black bear image

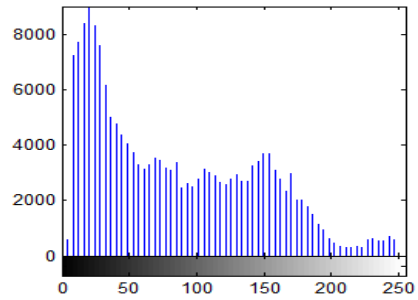

(b) Histogram

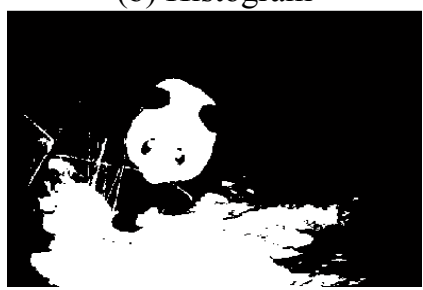

(d) Image segmentation 
Fig 4. Segmentation effect of Panda image

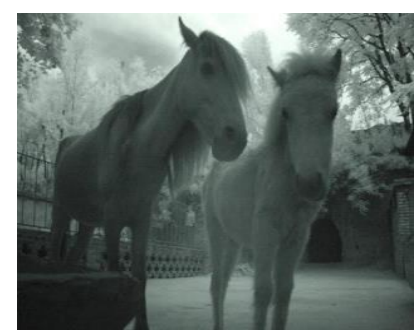

(a) Original image

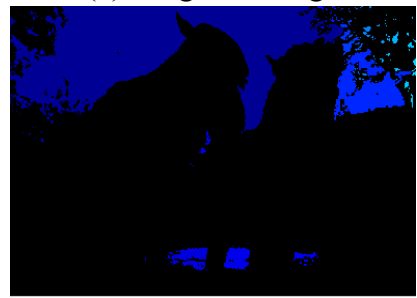

(c) Label image

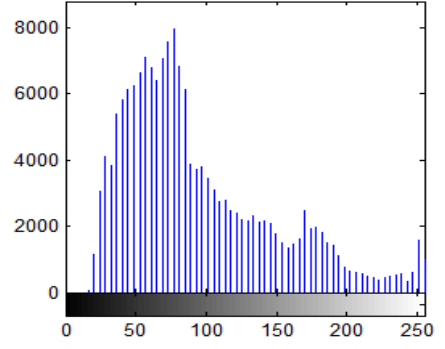

(b) Histogram

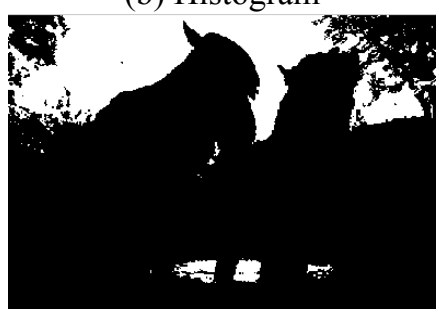

(d) Image segmentation
Fig 5. Segmentation effect of Horses image

From the above table data and test results, it can be seen that the background and target in the infrared of Aircraft and Black bear images is relatively simple and single, but the gray difference between the background and target is small, and the gray distribution of the background is uneven. Our proposed algorithm can divide the background into targets, it still can get the complete target, the segmentation result hasn't misclassification phenomenon.

In the infrared images of Panda and Horses, the gray difference between the target and the background is slightly larger, and the background is more complex. There are multiple targets in the infrared image and the gray level of the target is not uniform. The segmentation results of our proposed algorithm hasn't problems of false segmentation and weak edge leakage, and it is still able to segment a complete target, and there isn't a certain degree of false segmentation.

The algorithm in this paper can not only reduce the amount of computation, but also remove a large number of dark background in the lower limit and some bright targets in the upper limit. In the remaining pixels of the target or background, the proportion of the target and the background is similar. Combined with Otsu principle and de algorithm, the optimal segmentation threshold is automatically searched within this threshold range for infrared target image Automatic image segmentation. According to the characteristics of infrared image, this algorithm selects five goodness method indexes: isoperimetric ratio, edge gradient, average gray level of target, aggregation degree of target pixels and number of target pixels, and puts forward a comprehensive goodness evaluation criterion. Using this criterion, the performance of all segmentation algorithms and traditional threshold segmentation algorithms proposed in this paper are evaluated and analyzed. The experimental results show that the evaluation criterion can effectively explain the advantages and disadvantages of the segmentation algorithm, which is consistent with people's subjective evaluation and the effect of deviation method.

\section{CONCLUSION AND FUTURE WORK}

According to the image features, image segmentation technology divides it into a certain number of non-overlapping regions, and the characteristics of each region are different, and extracts the important information from each part of the image. It is a key technology in image processing and analysis, and it is also a typical difficulty because only after the segmentation is finished can the target recognition, classification and positioning be conducted. Infrared imaging technology is passive non-contact detection technology and its basic principle is to map the infrared radiation of the scene received by infrared detector into grayscale value and then get the visible image. The part with high radiation intensity is reflected as the region with high grayscale value in the image. This paper for the infrared detection means, has aimed to study the infrared target detection and segmentation algorithms under complex backgrounds, analyzed the infrared radiation characteristics of the target and background by learning the relevant theories of infrared image processing and image segmentation, pre-processed the infrared image based on the modeling of infrared image and achieved infrared target extraction and segmentation. This paper has integrated the excellent optimization ability of DE to improve the conventional OTSU image segmentation method, proposed the new-type segmentation and detection algorithm, and enhanced the real-time performance while ensuring the detection effect. The experiment has shown the effect and feasibility of the mixed algorithm proposed in this paper.

The limitations of this research and future directions: although this paper has done a lot of research on infrared target segmentation algorithm and its evaluation criteria, due to the complexity of the image and the uncertainty of the target, it is difficult to form a completely general method or index, which needs to be analyzed for more examples. The future research work can introduce the research on the segmentation algorithm of prior knowledge. The algorithm in this paper is based on the underlying data-driven and performs automatic segmentation without fully considering the prior knowledge. In fact, for specific targets, we can also obtain effective prior knowledge through multi sample training, and then combine the prior knowledge for image segmentation.

\section{ACKNOWLEDGMENT}

This work was supported by the Hebei Association for Science and Technology(Project Name: A New Mechanism and Model for the Integration of Production, Education, Research and Application).

\section{REFERENCES}

[1] Jones, M.; Lai, H.; McBride, C. , "Machine Learning Powered Parameter -Free 2D And 3D Image Segmentation and Object Analysis Pipeline", Molecular Biology of the Cell, vol.29, no.26, pp.38-39, 2018. 
[2] Jiang, Feng; Li, Na; Zhou, Lili, "Grain Segmentation of Sandstone Images Based on Convolutional Neural Networks and Weighted Fuzzy Clustering”, Iet Image Processing, vol.14, no.14, pp.3499-3507, 2020.

[3] Saha, Sudipan; Mou, Lichao; Qiu, Chunping, "Unsupervised Deep Joint Segmentation of Multitemporal High-Resolution Images", IEEE Transactions on Geoscience and Remote Sensing, vol.58, no.12, pp.8780-8792, 2020 .

[4] Liang, Luming; Zhang, Zhi-min, "Structure-Aware Enhancement of Imaging Mass Spectrometry Data for Semantic Segmentation", Chemometrics and Intelligent Laboratory Systems, vol.171, pp.259-265, 2017.

[5] Zhou, Zijian; Sanders, Jeremiah W.; Johnson, Jason M, "Met Net: Computer-aided Segmentation of Brain Metastases in Post-contrast T1-weighted Magnetic Resonance Imaging", Radiotherapy and Oncology, vol.153, pp.189-196, 2020.

[6] Reed I S, Gagliardi R M, Stotts L. "Optical Moving Target Detection with 3-D Matched, Filtering", IEEE Trans on AES, vol.24, no. 4, pp.327-335, 1998.

[7] Blostein S D, Huang T S. "Detecting Small Moving Object in Image Sequences Using, Sequential Hypothesis Testing", IEEE Trans on SP, vol.39, no.7, pp.1611-1629, 1991.

[8] Meng Hong, Yu Jiaxue, Qin Lei. "Infrared Image Segmentation Method of Electrical Equipment Based on Ca and Otsu", Power Automation Chemical Equipment, vol.31, no.09, pp.92-95, 2011.

[9] Banerjee, Abhirup; Maji, Pradipta, "Segmentation of Bias Field Induced Brain MR Images Using Rough Sets and Stomped-T Distribution", Information Sciences, vol.504, pp.520-545, 2019.

[10] Yang, Aqing; Huang, Huasheng; Zheng, Chan, "High-Accuracy Image Segmentation for Lactating Sows Using A Fully Convolutional Network", Biosystems Engineering, vol.176, pp.36-47, 2018.

[11] Aguiar, Gabriel Jonas; Mantovani, Rafael Gomes; Mastelini, Saulo M. , "A Meta-Learning Approach for Selecting Image Segmentation Algorithm", Pattern Recognition Letters, vol.128, pp.480-487, 2019.

[12] Tetard, Martin; Marchant, Ross; Cortese, Giuseppe, "Technical Note: A New Automated Radiolarian Image Acquisition, Stacking, Processing, Segmentation and Identification Workflow", Climate of the Past, vol.16, no.6, pp.2415-2429, 2020.

[13] Wang, Mingxu; Wang, Xingyuan; Zhang, Yingqian, "A Novel Chaotic Encryption Scheme Based on Image Segmentation and Multiple Diffusion Models", Optics and Laser Technology, vol.108, pp.558-573, 2018.

[14] Pedroso, Dorival M.; Bonyadi, Mohammad Reza; Gallagher, Marcus, "Parallel Evolutionary Algorithm for Single and Multi-Objective Optimisation: Differential Evolution and Constraints Handling", Applied Soft Computing, vol.61, pp.995-1012, 2017

[15] Zhao, Fuqing; Xue, Feilong; Zhang, Yi, "A Hybrid Algorithm Based on Self-Adaptive Gravitational Search Algorithm and Differential Evolution", Expert Systems With Applications, vol.113, pp.515-530, 2018

[16] Wang, Weiwei; Wu, Cuiling, "Image Segmentation By Correlation Adaptive Weighted Regression”, Neurocomputing, vol.267, pp.426-435, 2017

[17] Kotte, Sowjanya; Pullakura, Rajesh Kumar; Injeti, Satish Kumar, "Optimal Multilevel Thresholding Selection for Brain MRI Image Segmentation Based on Adaptive Wind Driven Optimization", Measurement, vol.130, pp.340-361, 2018.
[18] Mehrtash, Alireza; Wells, William M., III; Tempany, Clare M, "Confidence Calibration and Predictive Uncertainty Estimation for Deep Medical Image Segmentation", IEEE Transactions on Medical Imaging, vol.39, no.12, pp.3868-3878, 2020.

[19] Li, Yupeng; Cao, Guo; Yu, Qian, "Active Contours Driven by Non-Local Gaussian Distribution Fitting Energy for Image Segmentation", Applied Intelligence, vol.48, no.12, pp. 4855-4870, 2018.

[20] Carvalho, L. E.; Sobieranski, A. C.; von Wangenheim, A, "3D Segmentation Algorithms for Computerized Tomographic Imaging: a Systematic Literature Review", Journal of Digital Imaging, vol.31, no.6, pp.799-850, 2018

[21] Zareie, Mina; Parsaei, Hossein; Amiri, Saba, "Automatic Segmentation of Vertebrae in 3D CT Images Using Adaptive Fast 3D Pulse Coupled Neural Networks", Australasian Physical \& Engineering Sciences in Medicine, vol.41, no.4, pp.1009-1020, 2018.

[22] Zhao, Jing; Ren, Jinchang; Zabalza, Jaime, "Cognitive Seismic Data Modelling Based Successive Differential Evolution Algorithm for Effective Exploration of Oil-Gas Reservoirs", Journal of Petroleum Science and Engineering, vol.171, pp.1159-1170, 2018.

[23] Huang, Jun; Ma, Yong; Zhang, Ying, "Infrared Image Enhancement Algorithm Based on Adaptive Histogram Segmentation", Applied Optics, vol.56, no.35, pp.9686-9697, 2017.

[24] Yuzgec, Ugur; Eser, Mehmet, "Chaotic Based Differential Evolution Algorithm for Optimization of Baker's Yeast Drying Process", Egyptian Informatics Journal, vol.19, no.3, pp.151-163, 2018.

[25] Peng, Lu; Liu, Shan; Liu, Rui, "Effective Long Short-Term Memory With Differential Evolution Algorithm for Electricity Price Prediction", Energy, vol.162, pp.1301-1314, 2018.

[26] Baig, Muhammad Zeeshan; Aslam, Nauman; Shum, Hubert P. H, "Differential Evolution Algorithm As A Tool for Optimal Feature Subset Selection in Motor Imagery EEG", Expert Systems With Applications, vol.90, pp.184-195, 2017

[27] Muangkote, Nipotepat; Sunat, Khamron; Chiewchanwattana, Sirapat, "Rr-cr-IJADE: An Efficient Differential Evolution Algorithm for Multilevel Image Thresholding", Expert Systems With Applications, vol.90, pp. 272-289, 2017.

[28] Bai, Bing; Liu, Pei-Zhong; Du, Yong-Zhao, "Automatic Segmentation of Cervical Region in Colposcopic Images Using K-Means", Australasian Physical \& Engineering Sciences in Medicine, vol.41, no.4, pp.1077-1085, 2018.

[29] Wang B, Gao X, Tao D, et al., "A nonlinear adaptive level set for image segmentation", IEEE Transactions on Cybernetics, vol. 44, no.3, pp.418-428, 2014

[30] Chabrier S, Laurent H, Rosenberger C, et al., "Supervised evaluation of synthetic and real contour segmentation results", Proc. of the 14th European Signal Processing Conference, 2006, pp. 1-4.

\section{Creative Commons Attribution License 4.0 (Attribution 4.0 International, CC BY 4.0)}

This article is published under the terms of the Creative Commons Attribution License 4.0 https://creativecommons.org/licenses/by/4.0/deed.en_US 